\title{
Statistical approach to food sampling
}

\author{
R.Y. Wong ${ }^{1}$, N.S. Ferguson ${ }^{1} \&$ C.F. Clark ${ }^{2}$ \\ ${ }^{I}$ University of Strathclyde, Scotland. \\ ${ }^{2}$ Highland Council, Scotland.
}

\begin{abstract}
Although the U.K. food sampling network is well developed, the number of food poisoning incidents continues on an upward trend. Existing food sampling programmes used by the U.K. local authorities, where they exist, operate in a 'hit or miss' fashion. The use of small sample sizes is also common. This study examined the costs and benefits of current sampling programmes, and proposes an alternative using statistically validated approach to food sampling. Because sampling involves errors due to uncertainties and variations, a statistically validated sampling model was developed in an attempt to determine suitable sample sizes under various sample proportions that would also satisfy good normal approximation in order to reduce margin of error to a minimum. However, the model illustrated that the use of small sample size involves a large margin of error, particularly when sample proportion is small, and current sampling regimes were far from reaching the minimum requirement. Consequently, this may lead to erroneous conclusion. In the main, if sampling has an important part in food safety activities in terms of risk assessment, then central government support towards sampling and analysis cost is vital. Routine sampling can be undertaken collectively at a regional basis, and the cost may be split among local authorities.
\end{abstract}




\section{Introduction}

As food sampling is part of routine food surveillance activities, application of food sampling programmes may help local authorities to plan and determine the types and frequency of sampling carried out over a specified period. However, two fundamental questions must be addressed: (i) What should be the appropriate sample size? (ii) What conclusions can be drawn from the analytical results? To decide a representative sample from the entire food population within a limited budget and resources is an extremely difficult task faced by many local authorities. Coupled with the financial constraint is the lack of scientific evidence concerning many types of food contaminants. In particular, level of pathogen in foodstuffs that might lead to food poisoning incident. Solutions to these problems involve application of statistical concepts of population probability and sampling. However, concern was expressed that the introduction of statistical analysis would simply entail substantially more sampling than is currently done, and hence more cost because of its emphasis on statistical quality [1]. For this reason, the main objective of this paper is to examine the relationship between sample size and margin of error in order to obtain a good estimate of population proportion. The understanding of precision of estimate will help local authorities on planning their routine food sampling programmes.

\section{Statistical sampling theory}

Since it is not feasible or practical to examine an entire lot of food, sampling is required so that the results of analyses from the portion of the lot is represented by the sample are used to draw conclusions about the whole. Acceptability or rejection of the lot is normally based on the levels of food contaminants presented in the sample. It is essential to understand both real-world effects of food contaminants and on the design of food sampling. However, it is inevitable that uncertainty and variability are present in various forms and are often unpredictable in their behaviours. For example, the effect of a contaminant on a subject will vary for a number of reasons: (a) observation or measurement errors, (b) spatial or temporal effects (c) natural variability [1]. To understand relationships between a contaminant and the subject group in the face of such forms of uncertainty and variation requires probabilistic assessment of levels of uncertainty and statistical methods to infer and explain the relationships. In terms of microbiological contaminants, choice of sampling plan for a particular food or related product will depend on how well the microbiology of that food is understood.

The main reason of food sampling is to try to learn something about the population from which the sample was drawn. Since the sample taken is likely to form only a fraction of the population, and any conclusion drawn about the population is subject to chance error. Careful design of sampling methods can minimise the scale of such errors, but the chance of making errors still exists. The main inquisition is to examine the magnitude of the sampling error in order to reflect the likelihood of accuracy of such estimates. Based on the Central 
Limit Theorem, the variable $p$ (sample proportion) is approximately normally distributed, regardless of the distribution of the variable under consideration. With a statistically large sample size of 30 or more [2], approximation by normal distribution is feasible. The accuracy of the estimate can be evaluated by means of confidence-interval estimate for ${ }^{\wedge} p$. The confidence intervals for population proportion [3], as shown in eqn (1) is:

$$
100(1-\alpha) \% \text { C.I. for proportion }=\hat{p} \pm z_{\alpha / 2} \cdot \sqrt{\frac{\hat{p}(1-\hat{p})}{n}}
$$

The margin of error in estimating a population proportion by a sample proportion, as shown in eqn (2), is:

$$
\text { Margin of error, } E=z_{\alpha / 2} \cdot \sqrt{\frac{\hat{p}(1-\hat{p})}{n}}
$$

For a fixed sample size, a decrease in confidence level yields a smaller $z$-score and leads to a decrease in length of the confidence interval, and therefore increases the precision, and vice-versa. In order to improve the precision, it is necessary to decrease the length of the confidence interval. By doing so the confidence level has to be lowered as well. However, if the precision is to be improved by reducing the length of confidence interval but without affecting the confidence level, this can be accomplished by decreasing the margin of error.

\section{Determination of sample size}

One way of determining the required sample size is to first specify the margin of error and confidence level of a confidence interval in advance so that the specifications can be met. In real situation, the probability of some members in a population which possess a certain attribute (for example: level of pathogens above stated requirement) is often unknown. Also, there is a major concern when determining an appropriate sample size when $p$ is low since the margin of error $(E)$ can be affected significantly by $n$. In order to investigate the relationship between these two variables, graphs of $E$ were plotted against $n$ with various $p$ at $95 \%$ C.I. for population percentage. Since the application of normal approximation requires that sample size should be large (i.e. $n \geq 30$ ), therefore $n$ were selected between 30 to 300 with sample proportions from 0.5 to 0.001 plotted on the same graph. However, in order to ensure that normal approximation is reasonably good (i.e. near normal), there are certain criteria in which $n$ should comply to. In fact, there are different opinions regarding such criteria. Ross [4] suggested that normal approximation will be reasonably good for values of $n$ satisfying $n p(1-p) \geq 10$. In order to optimise the situation, this criterion was adopted for this research so that a reasonable area was chosen as 
Transactions on Biomedicine and Health vol 5, @ 2001 WIT Press, www.witpress.com, ISSN 1743-3525

230 Environmental Health Risk

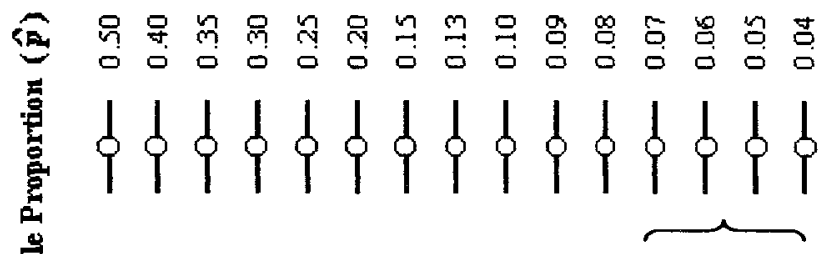

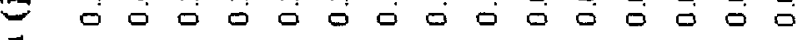

傿
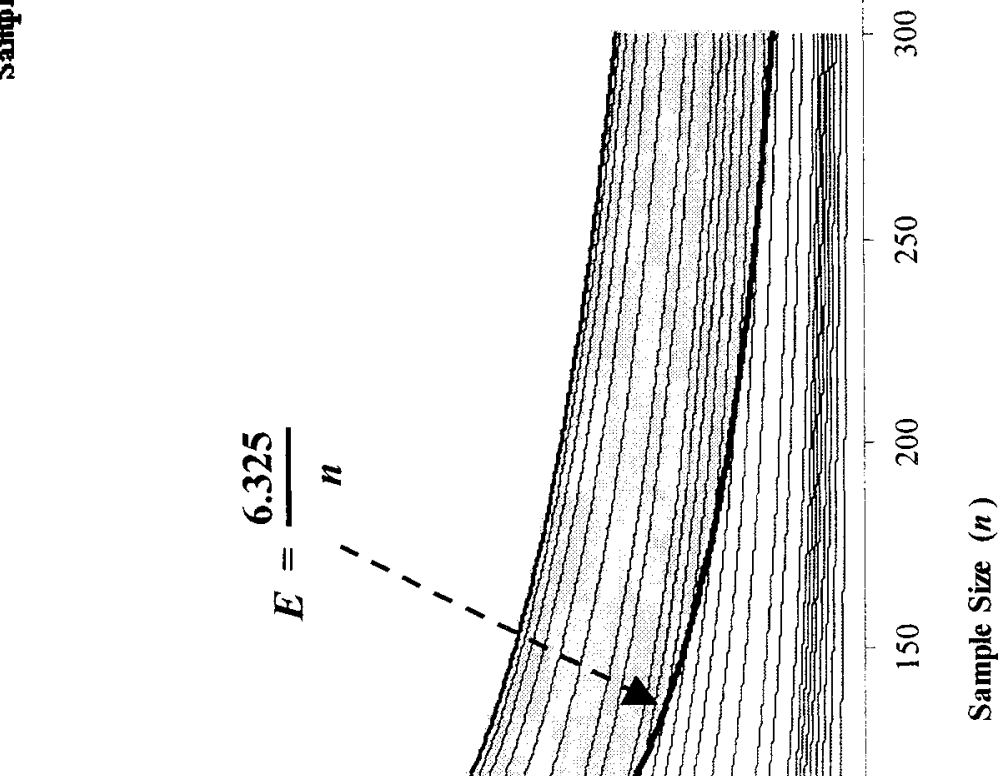

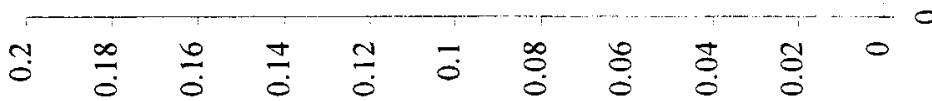

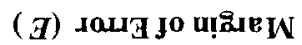

Figure 1: Illustration of suitable sample size at $95 \%$ C.I. that satisfied good normal approximation 
the requirement for near normality. Based on the requirement under this criterion, Figure 1 was plotted at $95 \%$ C.I. where the $z$-score for $95 \%$ C.I. is 1.96 .

$$
\left\{\begin{array}{l}
E=1.96 \sqrt{\frac{\hat{p}(1-\hat{p})}{n}} \\
n \hat{p}(1-\hat{p})=n
\end{array} \quad \Rightarrow \quad E=\frac{6.325}{n} \quad \text { (at } 95 \%\right. \text { C.I.) }
$$

The sample sizes $(n)$ satisfying good normal approximation for $95 \%$ C.I. was enclosed in the shaded area. From Figure 1, it was shown that more lines with lower sample proportion $(\hat{p})$ were excluded from the shaded area when sample size $(n)$ decreased. This exclusion became more apparent when $n$ was less than 100. For those with relatively larger $\hat{p}$, the negative gradient was very steep when $\mathrm{n}<50$. For those with relatively smaller ${ }^{2} p$ and/or larger $n$, the gradient gradually dropped and approached zero gradient. Negative gradient reflected that smaller sample size $(n)$ had greater margin of error $(E)$; and a decrease in gradient occurred when $n$ became larger, resulting with smaller $E$. Intersections between the line for good normal approximation and the curves of various sample proportions represented the minimum sample sizes to achieve good normal approximation. Taking $95 \%$ confidence level as a marker, the ratio of margin of error against sample proportion $\left(\mathrm{E} /{ }^{\wedge} p\right)$ was shown in Table 1 in order to illustrate the scale of sampling error in comparison with its sample proportion under minimum sample size $\left(n_{\min }\right)$ for good normal approximation.

Table 1: Ratio of $E / \hat{p} p$ at minimum sample size

\begin{tabular}{|c|c|c|c|}
\hline \multirow{2}{*}{$\begin{array}{c}\text { Min. sample size } \\
\left(n_{\text {min }}\right)\end{array}$} & $\hat{p}$ 95\% confidence intervals $(\%)$ & \multirow{2}{*}{ Ratio $(\mathrm{E} / \hat{p})$} \\
\hline & $\hat{p}$ & $E$ & 0.316 \\
\hline 40 & 50 & \pm 15.8 & 0.378 \\
\hline 42 & 40 & \pm 15.1 & 0.411 \\
\hline 44 & 35 & \pm 14.4 & 0.440 \\
\hline 48 & 30 & \pm 13.2 & 0.476 \\
\hline 53 & 25 & \pm 11.9 & 0.510 \\
\hline 62 & 20 & \pm 10.2 & 0.540 \\
\hline 78 & 15 & \pm 8.1 & 0.546 \\
\hline 89 & 13 & \pm 7.1 & 0.570 \\
\hline 111 & 10 & \pm 5.7 & 0.578 \\
\hline 123 & 9 & \pm 5.2 & 0.575 \\
\hline 138 & 8 & \pm 4.6 & 0.586 \\
\hline 154 & 7 & \pm 4.1 & 0.600 \\
\hline 178 & 6 & \pm 3.6 & 0.600 \\
\hline 211 & 5 & \pm 3.0 & 0.600 \\
\hline 264 & 4 & \pm 2.4 & \\
\hline & & & \\
\hline
\end{tabular}




\section{Discussion}

The main purpose of carrying out food sampling is to try to gain information about the food lot since it is not practically possible to examine the entire population. However, it is inevitable that sampling is subjected to both uncertainty and variation. It is thus important that these are taken account of within the sampling regime, otherwise the sampling results would be open to errors. Consequently, failure to acknowledge uncertainty and variation may lead to erroneous decisions based on sampling results. To overcome the problem of sample variation and achieve a higher confidence in estimating the population proportion, the determinant rests on sample size. A relatively larger sample size is more likely to yield a better result; but in practice, this is often hindered by financial and resource constraints. In fact, the true level of food contaminant at any location can not be known by sampling, unless the entire food lot is tested. But by means of statistical analysis, such variation can be quantified and a degree of confidence in which the true level lies can be obtained and justified. Therefore, it is aimed at selecting a minimum sample size to reduce the cost but at the same time obtaining a good estimate of the population proportion. This work examined the behaviour of margin of error $(E)$ under different sample size and sample proportion. In order to assure good normal approximation, it was based on the criterion for values of $n$ satisfying $n p(1-p) \geq 10$. The main purpose was to test the precision of the estimate from the level of $E$ at each of their sample proportions $(\hat{p})$.

One main problem was that curves with small sample proportions were excluded from good normal approximation unless sample sizes were very large. This could be argued that a sample size of 30 or above would satisfy the minimum requirement of normal approximation to sampling distribution. Strictly speaking, such statement might well not be considered as entirely untrue, but the main point was to obtain a reasonably good estimate of sampling error and confidence intervals through normal approximation from the sampling distribution graph in order to predict whether such sample would be a representative of the population. If the criterion for good normal approximation was not met, then such prediction might be inaccurate. Even if it was assumed that normal approximation was met, small sample proportion had many others problems for proper statistical analysis. As shown in Table 1, a decrease in sample proportion caused a significant increase in $E / p$ ratio. It is important to note that it is the $E /{ }^{\prime} p$ ratio that is crucial to determine whether such sample is statistically acceptable or not. In order to control the $E / p$ ratio and shorten the width of the intervals, a substantially large sample size would be required.

To ensure that a sample taken is a true representative of a food lot is neither simple nor obvious. It is extremely important that officers plan sampling carefully so that the samples taken are representative. One of the main difficulties is the detection of food contaminants at low levels, especially those which present a severe hazard to human health. No matter how low the probability of defective food units in the whole batch, it is important not to overlook this issue. In particular, pathogens such as Escherichia coli $\mathrm{O} 157$ or 
Clostridium botulinum [5] can spread and multiply rapidly, and lead to fatal incidents even at low level. This work indicated that a very large sample size would be required if sample proportion was low. However, current practice reflected that such sample size is not possible at all to be included in the food sampling programmes used by the local authorities. Indeed, when sample size is so small that normal approximation can not be met, then there is no confidence at all to justify the accuracy of the sample statistics.

Food Safety Act 1990 clearly stated that it is an offence to sell any food which fails to comply with food safety requirements if it is unfit for human consumption or rendered injurious to health, etc. [6]. The reason of food sampling and subsequent analysis is to collect some information about the foodstuffs sold in food premises whether compliance of agreed requirement is met. Such requirement may be set as legal standards, specifications or even guidelines; and sampling may be carried out routinely for the investigation of microbiological, chemical or physical contamination presented in food, as well as trading standard requirement on food labelling and composition. Apart from the need of national concern on food safety, European Commission also places a heavy burden on Member States on the submission of statistical returns. In order to illustrate competence in food law enforcement, Article 14 of the Official Control of Foodstuffs Directive (89/397/EEC) [7] requires Member States to submit details of the number and type of inspections and infringements as well as results of food samples to the Commission in an annual basis. Therefore, in order to comply with national and European food legislation, a lot of effort and resources are spent on routine sampling carried out mainly at local level. However, local authority reports [8] indicated that the use of small sample size is commonplace, with no application of statistical validation in the food programmes.

\section{Conclusion}

If food sampling is considered as a tool to assess risk, then based on the current food sampling regime, statistical theory of this model suggests that the use of small sample size involves a large margin of error, particularly when sample proportion is small. Consequently, this may lead to erroneous conclusion, and confidence of procuring representative sample to evaluate compliance with food safety legislation becomes questionable. Since sampling is a costly activity, it may not be an efficient and effective way to assess risk in food if precision cannot be obtained. In the main, the importance of sample size must not be overlooked in the existing sampling programmes, otherwise effort and resources could well be spent elsewhere to effectively prevent the occurrence of foodborne illness. In general, large sample size may not be feasible at local level due to the high cost, but small sample size would lead to erroneous conclusions. If local authorities consider undertaking routine sampling collectively at a regional basis, such high cost may be split. Central government support towards sampling and analysis cost is vital to the success of efficient and effective food sampling programme, and consequently lead to the prevention of food-borne illness. 
To determine a suitable sample size of any specific food contaminants in any particular food type, estimation of possible food contaminant levels in the lot (i.e. population proportion of defective items) would provide a clear picture of what $n$ should be for good approximation. Due to the fact that there are numerous food contaminants among large variety of foodstuffs, further research on the determination of critical levels for both chemical and microbiological food contaminants hazardous to health is necessary in order to improve the current situation. Without the existence of food standards agreed among the enforcing bodies, sampling itself becomes meaningless.

\section{References}

[1] Barnett, V. and O'Hagan, A., Setting Environmental Standards, Chapman \& Hall: London, 1997.

[2] Owen, F. and Jones, R., Statistics, Pitman Publishing Limited: London, 1986.

[3] Weiss, N.A., Elementary Statistics, Addison Wesley Longman: Harlow, 1999.

[4] Ross, S.M., Introduction to Probability and Statistics for Engineers and Scientists, John Wiley \& Sons: Singapore, 1987.

[5] Hobbs, B.C. and Roberts, D., Food Poisoning and Food Hygiene, Arnold Publishers: London, 1997.

[6] Bassett, W.H., Environmental Health Procedures, Chapman and Hall: London, 1995.

[7] Wong, R.Y., Requirements under the Official Control of Foodstuffs Directive (89/397/EEC), Environmental Health Scotland, 11(4), pp. 9-13, 1999.

[8] Scottish Office, Quarterly Returns of 19 Scottish Local authorities' Inspection Statistics - Official Control of Foodstuffs, Scottish Office Publication, 1991-94. 